\title{
DISTRIBUTIONS WITH COMPLETE MONOTONE DERIVATIVE AND GEOMETRIC INFINITE DIVISIBILITY
}

\author{
R. N. PILLAI* AND \\ E. SANDHYA, ${ }^{*}$ University of Kerala
}

\begin{abstract}
It is shown that a distribution with complete monotone derivative is geometrically infinitely divisible and that the class of distributions with complete monotone derivative is a proper subclass of the class of geometrically infinitely divisible distributions.
\end{abstract}

\section{Introduction}

The concept of geometric infinite divisibility (g.i.d.) was introduced by Klebanov et al. (1984). A random variable $Y$ is said to be g.i.d. if for every $p \in(0,1)$, there is a sequence of independently identically distributed (i.i.d.) random variables $X_{1}^{(p)}, X_{2}^{(p)}, \cdots$ such that

$$
p\left\{N_{p}=k\right\}=p(1-p)^{k-1}, \quad k=1,2, \cdots
$$

and $Y, N_{p}$ and $X_{j}^{(p)}(j=1,2, \cdots)$ are independent. (The symbol $\stackrel{\text { d }}{=}$ expresses equality of distributions.) They also established that a distribution $F$ with characteristic function (ch.f.) $f(t)$ is g.i.d. if and only if $\exp \{1-1 / f(t)\}$ is infinitely divisible (i.d.). Pillai and Sandhya (1990) have shown that the class of g.i.d. distributions is a proper subclass of i.d. distributions. An obvious example of g.i.d. distribution is Laplace which has ch.f. $1 /\left(1+t^{2}\right)$.

Every distribution considered here has positive support. A distribution $F$ is said to have complete monotone derivative (c.m.d.) if $(-1)^{\prime \prime} F^{(n)}(x) \leqq 0$ for $n \geqq 1$. Goldie (1967) has shown that a distribution with c.m.d. is i.d. Here we show a much stronger result than Goldie's that if a distribution has c.m.d., then it is g.i.d. It is stronger because of the result by Pillai and Sandhya mentioned above.

\section{Geometric infinite divisibility of distributions with complete monotone derivative}

Let us consider the following lemmas.

Lemma 2.1. A probability distribution $F$ has c.m.d. if and only if it is a mixture of exponentials, i.e., its Laplace transform $\mathscr{H}(\lambda)$ can be written in the form

$$
\mathscr{L}(\lambda)=\int_{0}^{\infty} \frac{x}{x+\lambda} d G(x),
$$

where the mixing distribution is $G(x)$.

Proof. Suppose $F$ has c.m.d. Then $\phi(x)=1-F(x)$ is completely monotone. Then, there

\footnotetext{
Received 16 November 1989; revision received 29 March 1990.

* Postal address: Department of Statistics, University of Kerala, Kariavattom (P.O.), Trivandrum-695 581, India.

The second author's research is supported by the Council of Scientific and Industrial Research, India in the form of a Junior Research Fellowship.
} 
exists a probability distribution $G(x)$ such that

$$
\phi(x)=\int_{0}^{\infty} e^{-u x} d G(u) .
$$

The Laplace transform of $F(x)$ is given by

$$
\begin{aligned}
\int_{0}^{\infty} e^{-\lambda x} d F(x) & =-\int_{0}^{\infty} e^{-\lambda x} d \phi(x) \\
& =-\int_{0}^{\infty} e^{-\lambda x} d\left(\int_{0}^{\infty} e^{-u x} d G(u)\right) \\
& =\int_{0}^{\infty} \frac{x}{\lambda+x} d G(x) .
\end{aligned}
$$

Conversely, if the distribution $F(x)$ is a mixture of exponentials, then

$$
F(x)=\int_{0}^{\infty}\left(1-e^{-u x}\right) d G(u)=1-\phi(x)
$$

and therefore $F(x)$ has c.m.d.

Lemma 2.2. Let $\phi(\lambda)$ be a finite mixture of exponentials:

$$
\phi(\lambda)=\sum_{j=1}^{n} p_{j} \frac{a_{j}}{a_{j}+\lambda}, \quad 0<a_{1}<a_{2} \cdots<a_{n} .
$$

Then $\phi(\lambda)$ is g.i.d.

Proof. By Steutel (1967)

$$
\phi(\lambda)=\frac{\prod_{j=1}^{n} \frac{a_{j}}{a_{j}+\lambda}}{\prod_{j=1}^{n-1} \frac{b_{j}}{b_{j}+\lambda}}
$$

where $0<a_{1}<a_{2} \cdots<a_{n}$ and $a_{j}<b_{j}, j=1,2, \ldots,(n-1)$.

$$
\begin{aligned}
& \frac{1}{\phi(\lambda)}=\frac{\prod_{j=1}^{n-1}\left(b_{j} /\left(b_{j}+\lambda\right)\right)}{\prod_{j=1}^{n}\left(a_{j} /\left(a_{j}+\lambda\right)\right)} \\
& \frac{1}{\phi(\lambda)}=\frac{\left(a_{1}+\lambda\right)\left(a_{n}+\lambda\right)}{a_{1} a_{n}} \frac{\prod_{j=1}^{n-1}\left(b_{j} /\left(b_{j}+\lambda\right)\right)}{\prod_{j=2}^{n-1}\left(a_{j} /\left(a_{j}+\lambda\right)\right)}
\end{aligned}
$$

$b_{1}<a_{2}<b_{3}<\cdots<a_{n-1}<b_{n-1}$.

Again by Steutel (1967)

$$
\frac{\left.\prod_{j=1}^{n-1}\left(b_{j} / b_{j}+\lambda\right)\right)}{\prod_{j=2}^{n-2}\left(a_{j} /\left(a_{j}+\lambda\right)\right)}=\sum_{j=1}^{n-1} \frac{p_{j} b_{j}}{b_{j}+\lambda} .
$$


Now

$$
\begin{gathered}
\frac{1}{\phi(\lambda)}=\frac{1}{a_{1} a_{n}} \sum_{j=1}^{n-1} \frac{p_{j} b_{j}\left(a_{1}+\lambda\right)\left(a_{n}+\lambda\right)}{\left(b_{j}+\lambda\right)}, \quad a_{1}<b_{j}<a_{n} . \\
\frac{\left(a_{1}+\lambda\right)\left(a_{n}+\lambda\right)}{\left(b_{j}+\lambda\right)}=\lambda+a_{1}+a_{n}-b_{j}+\frac{a_{1} a_{n}-b_{j}\left(a_{1}+a_{n}-b_{j}\right)}{\left(b_{j}+\lambda\right)} .
\end{gathered}
$$

The right-hand side of (2.2) has c.m.d. From Kingman (1964) and Yannaros (1988), $\phi(\lambda)$ is g.i.d. if and only if $\phi(\lambda)=1 /(1+\psi(\lambda))$, where $\psi(\lambda)$ has c.m.d. Therefore it follows that if $1 / \phi(\lambda)$ has c.m.d., then $\phi(\lambda)$ is g.i.d.

Theorem 2.1. A probability distribution $F$ with c.m.d. is g.i.d.

(We understand from the referee that this result has been proved by Olof Thorin in an unpublished paper, using an entirely different approach.)

Proof. By Lemma 2.1, $F$ is a mixture of exponentials. By Steutel (1967), any mixture of exponentials is the limit of finite mixtures of the form (2.1). By Lemma 2.2, any finite mixture is g.i.d. By Klebanov et al. (1984), the limit of g.i.d. distributions is again g.i.d.

Theorem 2.2. The class of distribution functions having c.m.d. is a proper subclass of the class of g.i.d. distributions.

Proof. We prove it via a counter-example. Consider the distribution with density

$$
f(x)=e^{-x} \int_{0}^{\infty}(\Gamma(u))^{-1} x^{u-1} e^{-u} d u
$$

with Laplace transform $1 /(1+\log (1+\lambda)), \lambda>0$, which is g.i.d. by Kingman (1964) and Yannaros (1988). To show that $f(x)$ is not completely monotone:

$$
\begin{aligned}
f^{\prime}(x) & =e^{-x} \int_{0}^{\infty}(\Gamma(u))^{-1} e^{-u} x^{u-2}[u-1-x] d u \\
& =\frac{e^{-x}}{x^{2}} \int_{0}^{\infty} \frac{u}{\Gamma(u)} e^{-u} x^{u} d u-(1+x) \frac{e^{-x}}{x^{2}} \int_{0}^{\infty} \frac{e^{-u} x^{u}}{\Gamma(u)} d u .
\end{aligned}
$$

The second integral is finite. Now,

$$
\int_{0}^{\infty} \frac{u}{\Gamma(u)} e^{-u} x^{u} d u=\int_{0}^{b} \frac{u}{\Gamma(u)} e^{-u} x^{u} d u+\int_{b}^{\infty} \frac{u}{\Gamma(u)} e^{-u} x^{u} d u=A+B,
$$

$\boldsymbol{A}$ is bounded

$$
\lim _{u \rightarrow 0} \frac{u}{\Gamma(u)} e^{-u} x^{u}=0
$$

By Stirling's approximation formula

$$
\Gamma(u) \approx \sqrt{2 \pi} u^{u-\frac{1}{2}} e^{-u}(1+r(u)) \text { where }|r(u)| \leqq e^{1 / 12 u}-1 .
$$

Therefore

$$
B=\frac{1}{\sqrt{2 \pi}} \int_{b}^{\infty} \frac{u x^{u} d u}{u^{u-\frac{1}{2}}(1+r(u))}
$$


Since $|r(u)| \leqq e^{1 / 12 u}-1$,

and

$$
\begin{aligned}
1-|r(u)| & \geqq 2-e^{1 / 12 u} \\
\frac{1}{1+r(u)} & \leqq \frac{1}{1-r(u)} \\
e^{1 / 12 u} & \leqq e^{1 / 12 b}
\end{aligned}
$$

Hence

Therefore

$$
2-e^{1 / 12 u} \geqq 2-e^{1 / 12 b} .
$$

$$
\frac{1}{1-|r(u)|} \leqq \frac{1}{2-e^{1 /(12 b)}}=a \text {. }
$$

Claim

$$
B \leqq \frac{a}{\sqrt{2 \pi}} \int_{b}^{\infty} \frac{u x^{u} d u}{u^{u-\frac{1}{2}}}
$$

Choose $b>x e^{\frac{7}{2}}$ so that (2.2) is satisfied, and

$$
\frac{u^{\frac{3}{2}} x^{u}}{u^{u}}<u^{-2}
$$

$$
u(\log u-\log x)>\frac{7}{2} \log u .
$$

$$
\int_{0}^{\infty} \frac{u}{\Gamma(u)} e^{-u} x^{u} d u<\int_{b}^{\infty} \frac{d u}{u^{2}}
$$

Therefore, when $u>(1+x), f^{\prime}(x)$ is positive and hence $f(x)$ is not completely monotone. Hence the theorem.

\section{Acknowledgement}

The authors are thankful to the referee for his valuable comments on this paper.

\section{References}

Goldie, C. M. (1967) A class of infinitely divisible distributions. Proc. Camb. Phil. Soc. 63, 1141-1143.

KIngman, J. F. C. (1964) On doubly stochastic Poisson processes. Proc. Camb. Phil. Soc. 60, 923-930.

Klebanov, L. B., Maniya, G. M. and Melamed, I. A. (1984) A problem of Zolotarev and analogs of infinitely divisible and stable distributions in a scheme for summing a random number of random variables. Theor. Prob. Appl. 4, 29, 791-794.

Pillai, R. N. and SandHya, E. (1990) On geometric infinite divisibility. To appear.

Steufel, F. W. (1967) Note on the infinite divisibility of exponential mixtures. Ann. Math. Statist. 38, 1303-1305.

Yannaros, N. (1988) The inverses of thinned renewal processes. J. Appl. Prob. 25, 822-828. 